\title{
48 week bone marker changes with Dolutegravir (DTG) plus Abacavir/Lamivudine (ABC/3TC) vs. Tenofovir/Emtricitabine/Efavirenz (EFV/TDF/FTC): the SINGLE trial
}

\author{
Y Yazdanpanah", M-A Khuong-Josses, L Hocqueloux, G Pialoux, J Durant, B Wynne, C Granier, P Tebas, K Pappa, \\ S Min
}

From International Symposium HIV and Emerging Infectious Diseases 2014

Marseille, France. 21-23 May 2013

\section{Background}

DTG is a once-daily integrase inhibitor that in combination with $\mathrm{ABC} / 3 \mathrm{TC}$, demonstrated superior efficacy with favorable tolerability over TDF/FTC/EFV (SINGLE study) at 48 weeks. EFV is associated with reduced Vitamin D levels through CYP450 enzyme induction; TDF has been associated with decreased bone mineral density (BMD). Antiretroviral treatment (ART) initiation is characterized by initial rapid decline in bone mineral density (BMD) that subsequently stabilizes. Bone turnover markers are increased during these BMD changes.

\section{Methods}

In the double-blind SINGLE study (table 1) we measured markers of bone turnover (bone-specific alkaline phosphatase, C-termial telopeptide type 1 collagen, osteocalcin, and procollagen type $1 \mathrm{~N}$-propeptide) \& Vitamin D at baseline (BL) and at 48 weeks. ANCOVA analyses were adjusted for the following factors age, sex, HIV RNA, CD4+ cell count, BL biomarker level, BMI, smoking status, and Vitamin D supplementation.

\section{Results}

833 subjects were analyzed: $84 \%$ males; $68 \%$ whites. Mean BL VL: $4.7 \log 10 \mathrm{c} / \mathrm{mL} ; 32 \% \mathrm{VL}>100,000 \mathrm{c} / \mathrm{mL}$ and mean BL CD4 cell count $=350$ cells $/ \mathrm{mm} 3$. Bone markers increased in both groups but the increases were significantly greater in subjects treated with TDF/FTC/EFV. Vitamin D levels decreased in both treatment groups; the differences were not significant between groups.

Table 1

\begin{tabular}{|c|c|c|c|c|}
\hline & $\begin{array}{l}\text { DTG+ABC/3TC change from } \\
\text { baseline* }(N=414)\end{array}$ & 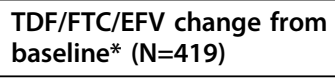 & $\begin{array}{l}\text { Geometric Mean } \\
\text { Ratio** }\end{array}$ & $\begin{array}{l}\text { [95\% } \mathrm{Cl} \text { for the } \\
\text { ratio] } \mathrm{p} \text {-value }\end{array}$ \\
\hline $\begin{array}{l}\text { Bone-specific alkaline } \\
\text { phosphatase }\end{array}$ & $15 \%$ & $60 \%$ & 0.720 & {$[0.685-0.756]<0.001$} \\
\hline $\begin{array}{l}\text { C-terminal telopeptide for } \\
\text { type } 1 \text { collagen }\end{array}$ & $33 \%$ & $68 \%$ & 0.789 & {$[0.747-0.832]<0.001$} \\
\hline Osteocalcin & $22 \%$ & $48 \%$ & 0.827 & {$[0.779-0.878]<0.001$} \\
\hline $\begin{array}{l}\text { Procollagen type } 1 \mathrm{~N}- \\
\text { propeptide }\end{array}$ & $30 \%$ & $66 \%$ & 0.784 & {$[0.749-0.821]<0.001$} \\
\hline $\begin{array}{l}\text { Vitamin D (25-hydroxy- } \\
\text { Vitamin D) }\end{array}$ & $-7 \%$ & $-10 \%$ & 1.036 & {$[0.970-1.107] P=0.292$} \\
\hline
\end{tabular}

*adjusted percent change from baseline $=100 \mathrm{X}$ adjusted geometric mean of (week 48/baseline)-100

${ }^{* *} \mathrm{GMR}=(\mathrm{DTG}+\mathrm{ABC} / 3 \mathrm{TC}+100) /(\mathrm{TDF} / \mathrm{FTC} / \mathrm{EFV}+100)$

Bichat Claude-Bernard Hospital - APHP, Paris, France 


\section{Conclusions}

After 48 weeks, significantly greater changes from baseline were observed for all bone markers in subjects receiving TDF/FTC/EFV, indicating more active bone turnover when compared to changes seen in subjects receiving DTG + ABC/3TC. These differences may correlate with known TDF-associated changes in BMD over time and further study of the potential advantages of a $\mathrm{DTG}+\mathrm{ABC} / 3 \mathrm{TC}$ regimen appear warranted.

Published: 23 May 2014

doi:10.1186/1471-2334-14-S2-P72

Cite this article as: Yazdanpanah et al: 48 week bone marker changes with Dolutegravir (DTG) plus Abacavir/Lamivudine (ABC/3TC) vs.

Tenofovir/Emtricitabine/Efavirenz (EFV/TDF/FTC): the SINGLE trial. BMC Infectious Diseases 2014 14(Suppl 2):P72.

Submit your next manuscript to BioMed Central and take full advantage of:

- Convenient online submission

- Thorough peer review

- No space constraints or color figure charges

- Immediate publication on acceptance

- Inclusion in PubMed, CAS, Scopus and Google Scholar

- Research which is freely available for redistribution

Submit your manuscript at www.biomedcentral.com/submit
C Biomed Central 